\title{
Prevención del daño: impacto económico y social
}

\author{
Damage prevention: \\ socioeconomic impact
}

\begin{abstract}
To prevent the damage, successful interventions were implemented to protect the child during the early years. As a result, $98 \%$ of children less than 5 years of age grew and developed according to WHO guidelines. This created a "domino effect" in older children reflected by the increased demands in education and health care followed by a period of rapid socioeconomic development. We can conclude that investment in prevention of undernutrition in the early years of life has been highly profitable. The example of Chile can be used as a model for other countries who still suffer from undernutrition and poverty.
\end{abstract}

Key words: brain damage, malnutrition, socioeconomic impact, prevention.

Las investigaciones Ilevadas a cabo entre los años 1950 y 1970, nos permitían afirmar que el medio ambiente adverso durante los primeros periodos de la vida, no sólo incrementaba los riesgos de enfermedades infecciosas y muertes prematuras, sino también dañaba el desarrollo físico e intelectual en forma permanente en los que sobrevivían, constituyendo un difícil obstáculo en la lucha contra la pobreza, la desigualdad y el desarrollo económico y social del país (1). De acuerdo a esta conclusión, la primera prioridad era prevenir tempranamente el daño de los que estaban naciendo, para preservar su salud y hacer posible la expresión de su potencial genético, tanto físico, como biológico, e intelectual. Todo ello con miras a lograr más adelante una adecuada inserción social en la demandante sociedad del conocimiento.

Debieron diseñarse intervenciones focalizadas y multifactoriales, dirigidas a proteger el normal crecimiento y desarrollo normal del niño durante los primeros años de vida, de modo que actuando simultáneamente, pudiesen prevenir los daños provocados tanto por la desnutrición temprana, como por otros factores ambientales adversos que frecuentemente acompañan a la pobreza. Como primera medida, debían satisfacerse los requerimientos nutricionales en las madres embarazada, al igual que los de sus hijos, y crear simultáneamente un ambiente adecuado para inducir el necesario apego madre-hijo, mediante la lactancia materna y la adecuada estimulación cognitiva, afectiva y verbal. Para ello era necesario establecer el control permanente y periódico del estado nutricional, tanto de la madre embarazada, como del niño después de nacer, hasta los 5 años de vida. Instruir a la madre en su cuidado, y prevenir en ellos las enfermedades infecto-contagiosas, mediante los programas de vacunación y la simultánea mejoría de las con-
Fernando Mönckeberg $B$.

Facultad de Medicina, Universidad Diego Portales, Santiago, Chile

Dirigir correspondencia a:

Dr.

Fernando Mönckeberg $B$.

Ejército 233, primer piso

Santiago Centro, Chile

Fono: 56-2-6768956

E-mail: fernando.monckeberg@udp.cl

Este trabajo fue recibido el 22 de Abril de 2014 y aceptado para ser publicado el 28 de Mayo de 2014.

diciones sanitarias del hogar, proveyéndolos de agua potable y adecuada eliminación de excretas. Como objetivo final, lograr el fortalecimiento de la estructura familiar, el cumplimiento de los roles parentales, con la adecuada exteriorización de las relaciones emocionales. Para más adelante, incrementar el nivel educacional y de escolaridad de los futuros padres $(2,3)$.

Fueron tanto las investigaciones realizadas en animales, como las observaciones en humanos, las que comprobaron que el retraso en el crecimiento físico e intelectual que se iba observando en edades posteriores (niñez, adolescencia y/o madurez), eran fundamentalmente debidos a secuelas de la adversidad medioambiental ocurridas durante los primeros años de vida. Teniendo presente estas conclusiones, el programa contemplaba dos intervenciones complementarias: "Prevención del daño durante los primeros años de vida" y "Tratamiento de los niños menores de dos años, con desnutrición grave. Las intervenciones de prevención fueron diseñados por el Instituto de Nutrición y Tecnología de los Alimentos (INTA) de la Universidad de Chile y entregado al Gobierno de la época (1974), él que las hizo suyas. Para su implementación, se creo a nivel de gobierno, un organismo denominado "Consejo Nacional para la Alimentación y Nutrición" (CONPAN), que agrupaba a varios ministerios y otros organismos estatales relacionados. El organismo lo presidía el ministro de salud y estaba dirigido por un Secretario Coordinador Ejecutivo, cargo que asumió el Dr. Fernando Mönckeberg, director de INTA.

Para el tratamiento de los niños con desnutrición grave, se creó una corporación privada, sin fines de lucro, denominada "Corporación para la Nutrición Infantil" (CONIN), la cual se responsabilizó del tratamiento y recuperación de los niños de 0 a 2 años de edad, que hubiesen llegado a una desnutri- 
ción grave, y que necesitasen de un tratamiento hospitalario especializado. De acuerdo al programa, tres instituciones tenían las siguientes responsabilidades: INTA (investigación y evaluación), COMPAN (ejecutor de intervenciones estatales de prevención) y CONIN (tratamiento de desnutridos graves). Ellas constituían una triada que debían trabajar en estrecha coordinación (3).

\section{PREVENCIÓN DEL DAÑO DURANTE LOS PRIMEROS AÑOS DE VIDA}

Antecedentes históricos. El año 1952, por ley se creó el Servicio Nacional de Salud, un organismo centralizado, cuya meta era la protección de la salud pública para toda la población chilena. Con esos fines, el año 1970, ya contaba con una infraestructura de salud consistente en 197 hospitales, 135 consultorios periféricos, y 942 postas rurales, con 120.000 funcionarios. En ella ya se había comenzado a desarrollar una atención materno-infantil preventiva, con énfasis principal en los programas de vacunación. El año 1970 su cobertura había alcanzado sólo al $60 \%$ de la población teóricamente asignada por la ley. A sus consultorios y postas rurales asistían las madres especialmente durante las campañas de vacunación o cuando los hijos presentaban alguna enfermedad infecto contagiosa, o de otra naturaleza. Se carecía de un programa preventivo, de control del "niño sano". Fue esta infraestructura de salud ya existente la que debió utilizarse como base para el inicio del programa de prevención de la desnutrición durante los primeros períodos de la vida: "Control médico de la madre embarazada y control periódico del crecimiento y desarrollo, hasta los cinco años de edad".

La primera prioridad fue completar la red de infraestructura para llegar a cubrir la población total asignada por la ley en las diferentes regiones del país. Para ello se debió comenzar con la construcción y habilitación de centros de salud, proveyéndolos con los equipos de profesionales debidamente entrenados y comprometidos, formado por médicos pediatras, obstetras, enfermeras universitarias, nutricionistas, asistentes sociales, personal auxiliar y de secretaría. Para ello fue necesario comenzar por coordinar la preparación en las universidades e institutos técnicos y profesionales de la época, con el objetivo de entrenarlos y capacitarlos en los programas y roles que debieran desempeñar. Este proceso demoró aproximadamente 10 años en llegar a cubrir el 100\% de la población asignada.

Desde un comienzo se comprobó que, dado el analfabetismo y la baja escolaridad de las madres, la mayor dificultad estaba en lograr que asistieran regularmente con sus hijos menores a los centros de salud, para el control del niño sano. Para atraerlas fue necesario implementar un incentivo suficientemente atractivo. Este fue la distribución gratuita de leche en polvo y otros alimentos en los Centros de Salud, no como donación sino como un derecho a todos los niños de 0 a 6 años. La entrega y distribución estaba condicionada al control periódico del niño sano que se realizaba en los centros de salud. Se estimó indispensable prestigiar la calidad de los productos, con el objeto de asegurar su aceptabilidad. Fue necesario desarrollar desde el comienzo una eficiente estrategia de marketing para los alimentos que se distribuían. Se esmeró en el control de calidad, presentación y envase del producto, asignándole el nombre comercial de "Leche Purita" (figura 1), y "Fortesan, Lechealim, según el caso (figura 2). Estos aportaban los requerimientos proteicos, vitamínicos y de elementos trazas, que satisfacían los requerimientos de los primeros seis años de edad. Para prestigiar su calidad, se desarrolló una estrategia consistente en exigir a las empresas productoras que se adjudicaban las licitaciones, otro requisito, cual era comercializar el mismo producto, con el mismo nombre e igual presentación, en el mercado establecido. La idea era que a través de ello, la madre conociera la aceptación del producto por grupos sociales de mayor nivel y que al mismo tiempo, se informara de su real valor comercial (5). Mediante este incentivo se consiguió crear el hábito de asistencia regular de la madre y su hijo a los centros de salud. En un comienzo era frecuente que las madres recibieran la leche y

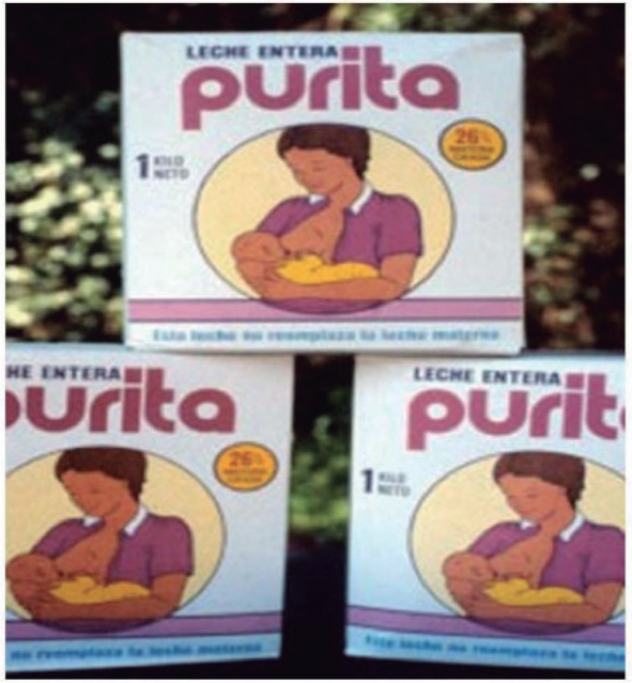


otros productos y conociendo su valor comercial, la vendieran a compradores instalados en las inmediaciones. Si bien esta conducta demoró algunos años en corregirse, se mantuvo su entrega, ya que el verdadero interés era conseguir la asistencia regular de las madres y sus hijos a los controles de salud. Con el tiempo, la madre iba adquiriendo una "cultura de salud" y se hacía responsable del cuidado de su hijo. Ello demoró algunos años, pero fue una estrategia útil para asegurar la asistencia regular de la madre, aprovechando para capacitarla en el cuidado del niño.

Intervenciones implementadas: Aún cuando en un comienzo no se contaba con una cobertura nacional de centros de salud en las diferentes regiones y localidades, los programas se iniciaron en los existentes, con las siguientes intervenciones (6):

a.- Control de la madre embarazada. El control médico del embarazo se inició a partir del quinto mes del embarazo y luego cada mes, hasta el momento del parto. Su asistencia a los controles se estimulaba con la entrega mensual de tres kilos de Leche Purita en polvo para la madre embarazada, con lo que se pretendía completar su requerimiento calórico y de nutrientes. El control se completaba con charlas educativas preparatorias para el parto y el posterior control del niño sano, asignándole la maternidad a la que debería asistir en el momento del parto.

Para las madres provenientes de poblaciones rurales alejadas, se organizó un programa, llamado del "Hogar de la madre campesina", consistente en una casa de acogida, anexa a la maternidad, donde la madre embarazada podía hospedarse desde días antes del parto y permanecer allí por algunos días después. Ese tiempo se aprovechaba para entregar información en el cuidado de recién nacido y especialmente en las técnicas de alimentación al pecho.

Con ello, al año de iniciado el programa, se había logrado que la totalidad de los partos fueran atendidos en maternidades y por personal profesional. Rápidamente comenzó a disminuir el porcentaje de niños que nacían con bajo peso (menos de 2.500 kilos), que disminuyó de 18\% a 3\% el año 1995 (figura 3).

b.- Control del niño sano. El programa diseñado contemplaba el control mensual durante los primeros seis meses y cada dos meses, hasta completar los dos años de edad. Posteriormente se completaba el control, cada tres meses, hasta los cinco años de edad. Este comprendía la enseñanza y estimulación de alimentación al pecho hasta los seis meses. Posteriormente, instrucciones de preparación de biberones, su higiene y alimentación complementaria, estimulación del apego materno infantil, enseñanza de técnicas de estimulación cognitiva, verbal y afectiva. Paternidad responsable y enseñanza de métodos de regulación de natalidad, dadas las elevadas tasas de fecundidad de la época (5,4 hijos por mujer en 1970). Prevención de enfermedades infecciosas, mediante el programa nacional de vacunas. En la actualidad el $100 \%$ de recién nacidos recibe la vacuna del BCG, el 95\% recibe la vacuna pentavalente (tres dosis) y el $92 \%$ la vacuna triviral.

En cada centro de salud, se organizó un registro mensual, centralizado, de peso y talla de todos los menores de cinco años en cada uno de los Centros de Salud, lo que permitió disponer mensualmente y en tiempo real, de la información del total de niños atendidos por regiones en el país. Esta información se centralizaba en las diferentes oficinas regionales de salud, las que la enviaban finalmente al Ministerio de Salud. De esta forma fue posible tener la información actualizada, de lo que estaba ocurriendo en todos y cada uno de ellos y en consecuencia tomar decisiones donde se detectaban deficiencias. En el periodo comprendido entre los años 1960 y 2000, disminuyeron los niños menores de 6 años con algún grado de desnutrición a $1.7 \%$.

c.- Tratamiento de los menores de dos años con desnutrición grave. Dada la gravedad y extensión de la desnutrición en los menores de dos años, fue también necesario implementar el tratamiento curativo a nivel nacional. La mayor parte de los niños menores de 6 años que presentaban retrasos en el crecimiento, su recuperación se lograba en forma ambulatoria

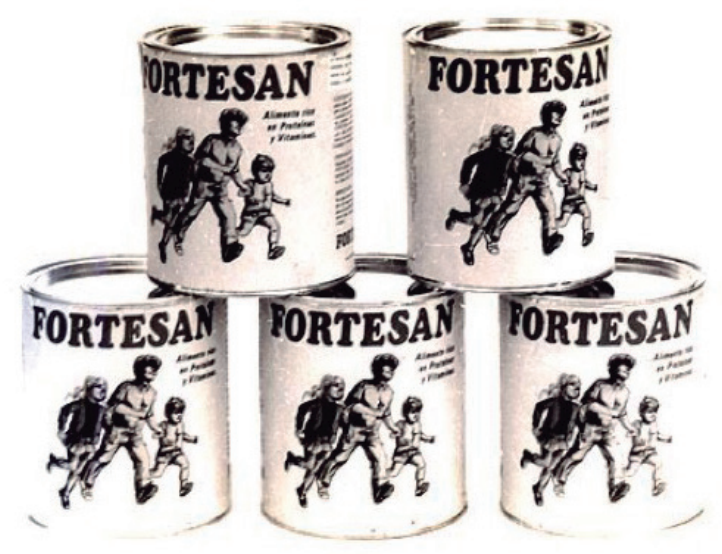

Presentación alimento en polvo infantil, distribuido por el Ministerio de Salud 
en los centros de salud. Con todo, algunos de ellos, especialmente hijos de madres adolescentes, fue necesario establecer un programa especial, de los llamados "Jardines Infantiles", donde el niño permanecía durante el día recibiendo el cuidado y la alimentación con el total de sus requerimientos nutritivos y calóricos (ver más adelante). Un número menor, durante el primero o segundo año de vida, llegaba a una desnutrición avanzada, que por su gravedad requería de hospitalización.

Hasta 1970, los niños menores de dos años con desnutrición grave abarrotaban las camas en los hospitales pediátricos, donde frecuentemente presentaban enfermedades intercurrentes con una elevada mortalidad. Por otra parte, la escasez de camas pediátricas, obligaba a altas prematuras, cuando recién iniciaban la recuperación. En sus casas, su destino era precario. Fallecían o volvían al hospital, hasta morir en algunos de estos lugares. Según nuestras evaluaciones, un niño con desnutrición avanzada, durante los primeros meses de vida, tenía $80 \%$ de posibilidad de fallecer antes de cumplir un año de edad, cualquiera fuese su tratamiento. La mortalidad intrahospitalaria de los menores de dos años con desnutrición avanzada, era de $30 \%$. Ello demostraba que el hospital convencional no era el lugar adecuado para el tratamiento, ya fuera por los altos índices de contaminación de enfermedades bacterianas o virales, como por las inconvenientes para desarrollar programas de estimulación afectiva y cognitiva personales, necesarios para su recuperación (7). Tampoco en el hospital convencional era fácil la incorporación de la madre al tratamiento y menos su participación en los programas educativos.

Después de un programa piloto exitoso, en un centro piloto, con lactantes desnutridos graves, menores de dos años de edad, se decidió extenderlo a todo el país. Para ello se creó una fundación privada, sin fines de lucro, llamada Corporación para la Nutrición Infantil (CONIN). Su objetivo era crear una red de pequeños centros hospitalarios distribuidos en las distintas regiones y ciudades, con el objeto de disminuir las infecciones intrahospitalarias observadas en el hospital pediátrico convencional y asegurar un tratamiento más personalizado, con estimulación psico-afectiva personal y grupal. De este modo, con el apoyo de la comunidad se creó un programa de pequeños centros pediátricos hospitalarios (con 50 a 60 camas cada uno, para niños menores de dos años) provistos con las facilidades necesarias, tanto de infraestructura física, como de profesionales, para tratar y recuperar niños con desnutrición grave en ese rango de edad. El Servicio Nacional de Salud, enviaba a CONIN los niños menores de dos años con desnutrición avanzada, ya fuera desde los hospitales pediátricos o los detectados en los centros de atención primaria. Entre los años 1975 y 1980, se crearon 34 pequeños centros hospitalarios con un total de 1700 camas, distribuidas en las diferentes ciudades (8). Cada centro tenía un director, médicos pediatras y un equipo profesional constituido por enfermeras universitarias, nutricionistas, educadoras de párvulos, kinesiólogos, y trabajadoras sociales, todos a tiempo completo cubriendo las 24 horas y de acuerdo a un programa previamente diseñado. Contaba además con 36 auxiliares de enfermería, que trabajaban en turnos de 8 horas y un equipo de voluntarias (entre 30 a 180 en cada centro), que colaboraban en los programas de estimulación individual y grupal. Cuando era posible, se incorporaban también las madres al tratamiento y recibían cursos de cuidados y estimulación de sus hijos.

El programa y las actividades fueron las siguientes: a.- Alimentación a base de leche de vaca y otros alimentos preparados bajo la estricta supervisión de la nutricionista. b.- Estimulación cognitiva y psico-sensorial, supervisada por la educadora de párvulos, de acuerdo con pautas previamente elaboradas. c.- Fisioterapia, supervisada por el especialista. d.- Estimulación afectiva, desarrolladas por las auxiliares de enfermería y voluntarias. e.- Incorporación de la madre al cuidado y estimulación del niño. f.- Educación de la madre en las técnicas de alimentación y nutrición, cuidado y apego del niño. g.- Programa de planificación familiar e incorporación a los programas de control del niño sano (7).

Ya los primeros resultados fueron muy exitosos observándose una disminución notable de las infecciones intercu-

FIGURA 3

TASAS DE MORTALIDAD INFANTIL (por cada 1000 nacidos vivos) Y PORCENTAGES DE RECIÉN NACIDOS DE BAJO PESO

(1060-2004)

\begin{tabular}{ccc}
\hline AÑOS & $\begin{array}{c}\text { TASA } \\
\text { MORTALIDAD } \\
\text { INFANTIL }\end{array}$ & $\begin{array}{c}\text { \% RECIÉN NACIDO } \\
\text { BAJO PESOS } \\
(-2,5 \mathrm{kgs})\end{array}$ \\
\hline 1960 & 120.2 & 19.2 \\
1965 & 101.3 & 16.3 \\
1970 & 82.1 & 13.2 \\
1975 & 55.4 & 11.6 \\
1980 & 31.8 & 8.2 \\
1985 & 19.7 & 7.0 \\
1990 & 16.8 & 6.4 \\
1995 & 12.3 & 6.1 \\
2000 & 8.9 & 5.2 \\
2004 & 7.8 & 3.8 \\
\hline
\end{tabular}


rrentes, un rápido inicio de la recuperación y una disminución notable de la mortalidad intrahospitalaria (menos del 1.5\%). Durante el período comprendido entre los años 1975 y 1995 , se recuperaron (peso para la talla) 82 mil niños desnutridos graves menores de dos años de edad (8) (figura 4). El programa impactó directamente sobre las tasas de mortalidad infantil, que descendieron de 150 por mil en 1950 a 8 por mil en 1990 (figura 3). Sin embargo, por lo general no se alcanzaba a recuperar la talla para la edad, pero si el peso para la edad, por lo que los niños se veían saludables, pero con déficit de talla. En la actualidad, habiendo prácticamente desaparecido los niños con desnutrición (0.2\% en 1995), CONIN se ha estado adaptando a la nueva realidad, disminuyendo el número de centros de 33 a sólo 8, con 200 camas, y reorientando su que hacer al tratamiento de niños menores de dos años, con necesidades nutritivas crónicas, (enfermedades metabólicas) y necesidades de alimentación compleja.

d.- Jardines Infantiles. Más allá de las intervenciones señaladas, existían realidades en que no era posible contar con la participación de la familia para asegurar el crecimiento y desarrollo del niño (madres adolescentes, niños no deseados, o problemas sociales complejos, pobreza extrema). Para ellos fue imprescindible implementar un sistema de atención directa, con el objeto de asegurar tanto los requerimientos nutricionales, como la necesaria estimulación emocional y afectiva. Para ello a partir de 1978 se comenzaron a crear los llamados "Jardines Infantiles", en los que los niños recibían diariamente el total de sus requerimientos nutritivos, en tres comidas diarias, además de programas de estimulación psico-afectiva y educacional, entregados por educadoras de párvulos adecuadamente capacitadas. Durante los primeros cuatro años se crearon doscientos cincuenta jardines infantiles, en que cada uno albergaba un promedio de sesenta niños. En los años sucesivos, cuando ya los desnutridos, menores de seis años, prácticamente desaparecieron, se incorporó el programa al sistema de educación pre-escolar, el que en la actualidad atiende a 600.000 mil preescolares en 5.700 establecimientos.
Más recientemente se ha comenzado a implementar salas cunas para niños menores de dos años, destinadas a satisfacer las necesidades de madres que trabajan fuera del hogar. Todo ello bajo el control del Ministerio de Educación.

e.- Alimentación escolar. Uno de los principales factores limitantes en la prevención de la desnutrición han sido el analfabetismo y la baja escolaridad de los padres. Para corregir estas deficiencias educacionales en los futuros padres, y a su vez revertir las elevadas tasas de deserción escolar, fue necesario buscar un incentivo para que los padres enviaran sus hijos a la escuela, para lo cual se desarrolló un programa de alimentación escolar. En la actualidad las escuelas disponen de comedores y cocinas adecuadamente equipadas, entregando, a lo largo del año, diariamente desayunos y almuerzos a un millón doscientos mil niños. Los servicios son contratados por empresas alimenticias especializadas, que abastecen y atienden los comedores. Ello bajo la dependencia del Ministerio de Educación, quien dicta las normas nutricionales y controla su cumplimiento.

f.- Saneamiento ambiental. Otro factor condicionante de la desnutrición era el inadecuado saneamiento ambiental de la mayor parte de la población, tanto urbana como rural. Hasta 1970, sólo el 74\% de la población urbana disponía de agua potable en su casa y apenas el $40 \%$ de ellas estaba conectada a un sistema adecuado de eliminación de excretas. Los porcentajes eran aun más deplorables en la población rural. Tal situación afectaba directamente la salud y nutrición durante los primeros años de vida, tanto por la elevada frecuencia de diarreas infecciosas, como por las alteraciones de la mucosa intestinal (9) que interfieren en la absorción de diversos nutrientes. Por ello debió iniciarse un extenso programa de saneamiento ambiental, tanto urbano, como rural. Se inició con la construcción de las llamadas casetas sanitarias que contaban con un baño, una cocina y un lavadero adosado a cada casa. Durante los primeros cuatro años, se construyeron 650.000 de estas unidades provistas de agua potable y conexión con alcantarillas (10). Posteriormente ello fue reemplazado por el

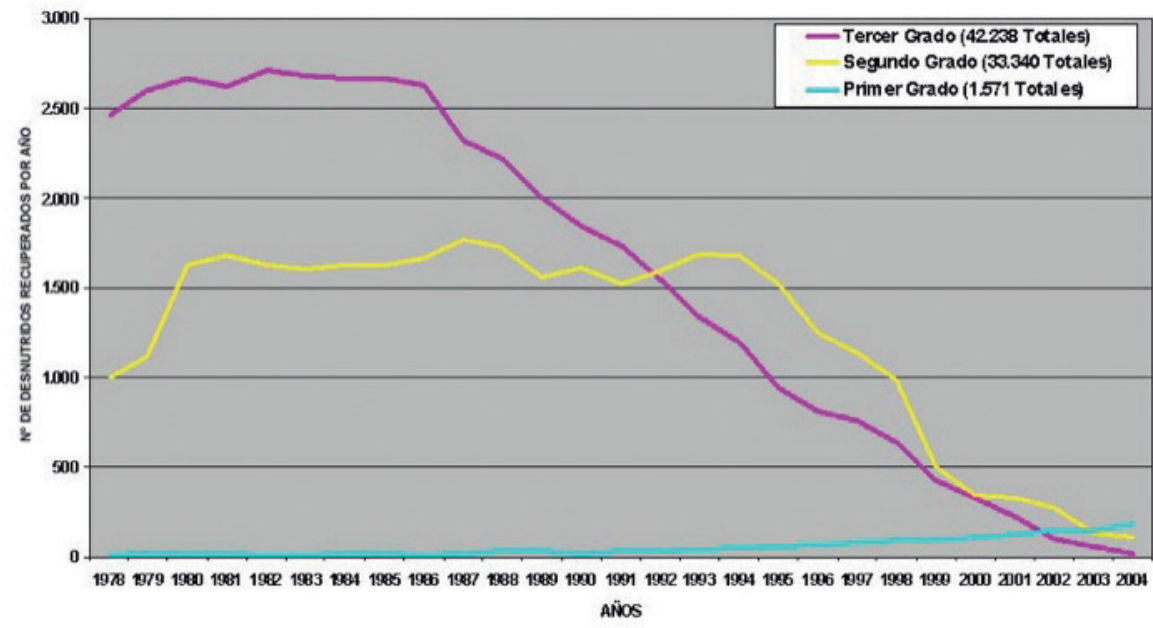


programa de construcción de viviendas sociales definitivas, conectadas a servicios de agua potable y alcantarillado. Dieciocho años más tarde (1985), el 99\% de la población urbana disponía de agua potable en la casa y el 83\% tenían conexión con la alcantarilla (figura 5). En la población rural, el porcentaje alcanzaba al $83 \%$ y $75 \%$ respectivamente. La mejoría de las condiciones sanitarias disminuyeron notablemente las muertes por enfermedades diarreicas (figura 6) En la actualidad se ha completado el saneamiento ambiental, tanto urbano, como rural. Al mismo tiempo que se ha desarrollado el programa de tratamiento de las aguas servidas de las diferentes ciudades, habiendo ya completado el proceso para el $85 \%$ del total de las aguas servidas del país.

\section{PREVENCIÓN DEL DAÑO Y "EL EFECTO DOMINÓ"}

Con la prevención del daño en los que iban naciendo, se fue incrementando la talla promedio, en la medida que iban alcanzando las subsiguientes edades (niñez, adolescencia y madurez). Con posterioridad comenzaron a evidenciarse progresivos cambios sociales y económicos: disminución de la

\section{Porcentaje de cobertura de agua potable y alcantarillado en zonas urbanas}

Chile, 1965-2005

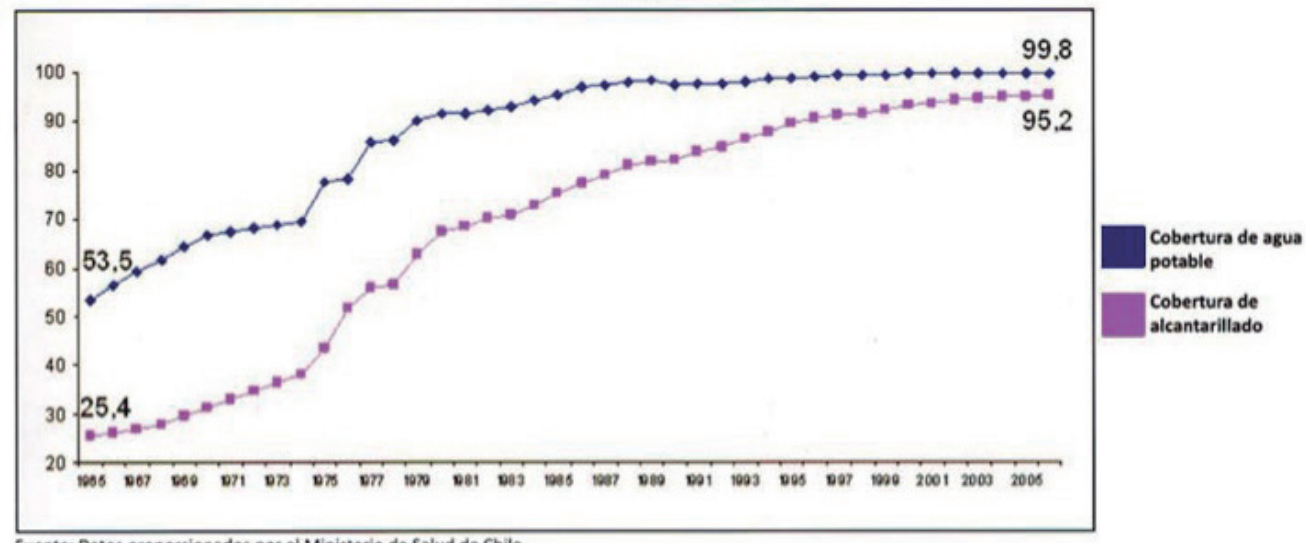

FIGURA 6

Muertes en menores de 1 año, por enfermedades respiratorias y diarreas 1960 - 2000 (por 100.000 nacidos vivos)

\begin{tabular}{ccc}
\hline Año & $\begin{array}{c}\text { Enfermedades } \\
\text { respiratorias }\end{array}$ & $\begin{array}{c}\text { Enfermedades } \\
\text { diarreicas }\end{array}$ \\
\hline 1960 & 4.318 & 1.365 \\
1970 & 2.793 & 1.476 \\
1980 & 502 & 210 \\
1990 & 161 & 36 \\
2000 & 86 & 12 \\
& & \\
\hline \multirow{2}{*}{ *Fuente: Informe anual, 2000 (Santiago, Chile) Ministerio de Salud } & \\
\end{tabular}


pobreza, desarrollo y consolidación de una clase media consolidada y un sostenido crecimiento económico. La secuencia observada en estos hechos, hace pensar efectivamente en la existencia de una relación de "causa efecto", entre la prevención precoz del daño nutricional en los que estaban naciendo por un parte, y los cambios sociales y económicos por otra.

Impacto en el desarrollo intelectual. En el año 1970, existía una elevada deserción escolar durante la educación básica, que alcanzaba un 60\%. Investigaciones de aquella época demostraron que ella era debida principalmente a dificultades en el aprendizaje (11), la que a su vez era consecuencia del daño cerebral producido por la desnutrición temprana (1). Existía además una relación, estadística significativa, entre el bajo rendimiento escolar y el menor diámetro del cráneo (12), como también entre el déficit de talla para la edad y el cociente intelectual.

A los pocos años de implementados los programas de prevención de la desnutrición, la deserción escolar comenzó a disminuir notablemente, de modo que ya en el año 1985, el $88 \%$ de los niños estaba terminando la educación básica (1992). Del mismo modo, comenzó a disminuir el déficit de talla en los menores de seis años, siendo Chile en la actualidad el país de América Latina con menor porcentaje de niños (menores de cinco años) con déficit de talla (figura 7). Ello fue seguido por un progresivo incremento en la demanda de la educación media, la que hasta 1970, sólo accedía el 15\% del grupo etario correspondiente. El Ministerio de Educación, ante la imposibilidad de dar respuesta al nuevo escenario, de un brusco incremento de la demanda educacional, abrió las posibilidades que se incorporara el sector privado en los niveles de educación básica y secundaria. Mediante un decreto con fuerza de ley, dio inicio a la llamada Educación Privada Subvencionada, por el que el estado compartía los costos educacionales en escuelas y colegios construidos y habilitados por el sector privado. Ello se tradujo en un rápido incremento en el número de escuelas privadas subvencionadas, de modo que en poco tiempo estas superaron las matrículas del sistema educacional estatal municipalizado. En la actualidad, la suma de ambas está logrando que el $70 \%$ del grupo etario correspondiente, esté terminando la educación media. De este modo la escolaridad promedio se incrementó progresivamente de 2 años en 1960, a 12 años en el 2010. (Ministerio de Educación 2012).

A su vez, el incremento de la educación media repercutió sobre la demanda de educación superior, que tampoco pudo ser satisfecha por la infraestructura de la educación estatal superior existente hasta ese entonces. De nuevo el Estado, mediante otro decreto ley, autorizó la existencia de universidades privadas sin fines de lucro. En pocos años se crearon 35 nuevas universidades. En 1950 el 5\% del grupo etario correspondiente tenía acceso a la educación universitaria, mientras que en la actualidad está ingresando a ella el $45 \%$. Sin embargo se ha puesto en evidencia una nueva problemática, relacionada con la calidad de la educación en los diferentes niveles.

Impacto en salud. Con la prevención de la desnutrición de los primeros años de vida, no sólo se ha logrado disminuir la mortalidad temprana, sino también han cambiado fundamentalmente los indicadores de salud. La mortalidad infantil es ahora de 7 por mil nacidos vivos, la más baja de América. Del mismo modo disminuyeron las muertes prematuras en los menores de 15 años, de un 52\% en 1951, a 3\% en la actualidad, con lo que se elevó la expectativa de vida de 38 años en 1950, a 80 años en la actualidad. Del mismo modo, han disminuido notablemente las enfermedades infectocontagiosas, algunas hasta casi desaparecer. Ello debido tanto a la disminución de la desnutrición infantil, que ha permitido restablecer la eficiencia del deprimido sistema inmunológico (tanto innato, como adaptativo), como a la mejoría de las condiciones sanitarias del hogar (agua potable, alcantarillas y tratamiento de aguas servidas), junto a la amplia cobertura alcanzada en los programas de vacunación. Habiendo disminuido las muertes prematuras han disminuido la necesidad de hospitalizaciones, pasando la atención pediátrica a ser una especialidad preponderantemente preventiva y ambulatoria, con el consiguiente ahorro de costos. Ahora los requerimientos

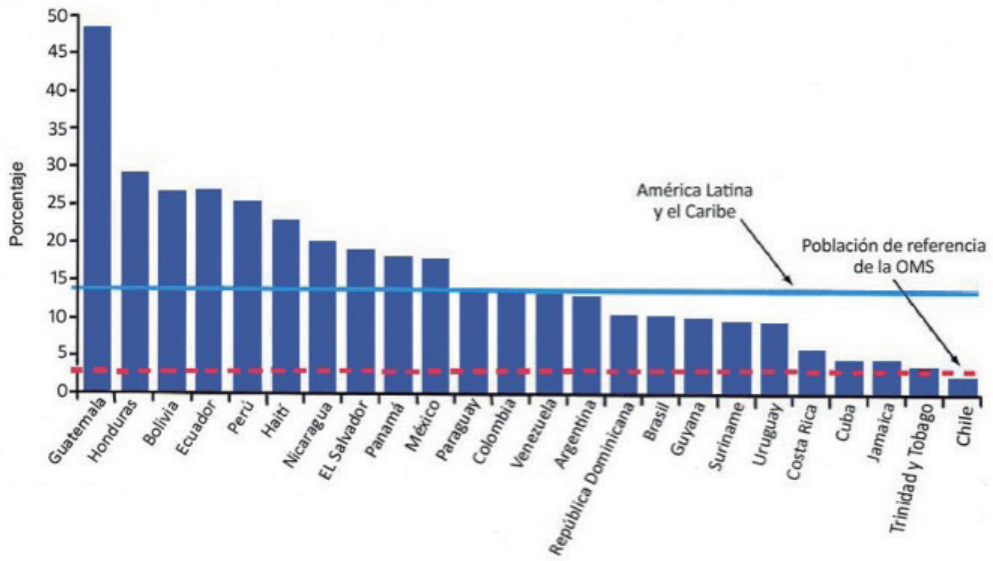

Fuente: Organización Mundial de la Salud, Comité Permanente de Nutrición. Quinto informe sobre la situación mundial de nutrición. OMS; 2004. Mother/Child Demographic, Health and Nutrition Surveys. 
de atención médica propiamente tal, son de control del niño sano, detección y tratamiento de enfermedades genéticas y metabólicas, trastornos alérgicos, enfermedades nutricionales específicas y obesidad.

Es muy posible que a futuro, se observen cambios en las enfermedades degenerativas propias de las edades de adultos. Son numerosas las observaciones científicas que observan que la desnutrición pre y post natal, incrementa la susceptibilidad a enfermedades como la obesidad, la hipertensión arterial, las enfermedades cardiovasculares, la diabetes tipo 2 y las dislipidemias $(13,14)$. Es probable que como consecuencia de la desaparición de la desnutrición precoz, estos indicadores también lleguen a modificarse favorablemente.

Impacto en el desarrollo físico. Es un hecho que el crecimiento en talla que alcanza una población, está relacionada con su alimentación, especialmente durante los primeros periodos de la vida. Una dieta deficiente durante los primeros periodos de la vida, condiciona un menor crecimiento, que se mantiene en edades posteriores, aun cuando mejore la alimentación. Lo mismo ocurre en animales de experimentación. Ratas subalimentadas durante los primeros diez días de vida, detienen su crecimiento. Posteriormente al ser realimentadas normalmente, lo reanudan, pero persistiendo por debajo normal, llegando a la edad adulta con menor talla. Lo mismo ocurre en cerdos subalimentados durante el primer mes de vida (15). Poblaciones humanas que se han estimado genéticamente como de baja estatura, la incrementan significativamente si mejoran su alimentación, especialmente durante los primeros periodos de la vida. Ello se ha observado en casi todos los países de Europa Occidental después de la Revolución Industrial. Así por ejemplo, Holanda en el año 1860, su población tenía una talla promedio de 1.63 metros. En 1990, esta se había elevado a 1.84 metros, lo que se atribuye a una mejor nutrición durante los primeros años de vida (16).

En Chile el año 1950, el déficit nutricional afectaba a un alto porcentaje de madres embarazadas, lo que se traducía en el menor peso del recién nacido. El 20\% de los recién nacidos pesaba menos de 2.5 kilos, con una menor talla. Durante los primeros 6 años de vida, el percentil 50 de talla del niño chileno, estaba bajo el percentil 3 de los niños de los Estados Unidos (17). Al haber logrado prevenir el daño de los primeros períodos de la vida, el año 2002 el porcentaje de bajo peso al nacer se había reducido a 3\%, y la talla promedio para la edad en los menores de seis años alcanzó la normalidad, destacándose dentro de todos los países de America Latina (18) (figura 7). Los adolescentes entre 10 y 18 años, en el periodo comprendido entre los años 1968 y 1988 (1.350.000 escolares), incrementaron también su estatura en un promedio de 10 centímetros (figura 8) (20). Actualmente $80 \%$ de los adultos jóvenes son significativamente más altos que sus padres.

\section{CONCLUSIONES}

Al prevenir el daño nutricional durante los primeros períodos de la vida, se ha logrado que un mayor porcentaje de los que van naciendo, expresen su potencial genético, tanto físico como intelectual. Por ello, Chile cuenta hoy con una población diferente, lo que comenzó a evidenciarse ya en los años 1980s. Más tarde, en la medida que los "no dañados" fueron alcanzando mayor edad, se fue exteriorizando un evidente progreso en el desarrollo económico y social. Mirando el proceso retrospectivamente, se hace difícil imaginar que Chile con el recurso humano que contaba en los años 1950s, hubiese podido tener éxito en el proceso de inserción económica internacional, como realmente ha sucedido.

Sin embargo, aún persiste un porcentaje importante de niños dañados precozmente, ya no por desnutrición, sino por factores socioculturales adversos que frecuentemente acompañan a la pobreza, que aún afecta alrededor del 14\% de la población. Robert Fogel, economista de la Universidad de Harvard, señala que: "la salud de una generación, a través de la experiencia de la madre, el lactante, el adolescente, influye en la fortaleza, salud, y longevidad de la generación siguiente, de modo que esta es más fuerte y vive mas, lo que

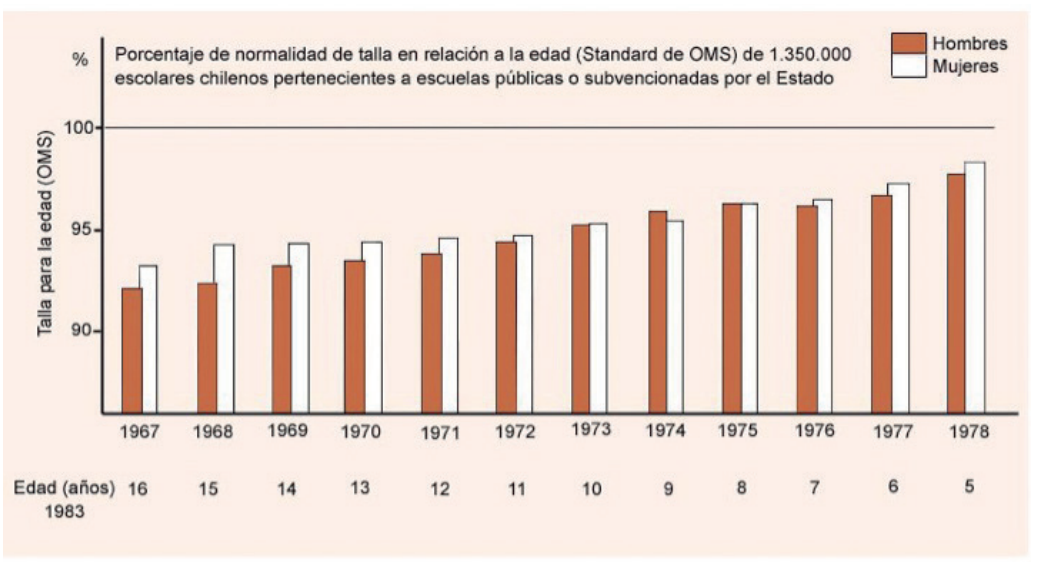

Déficit de talla de escolares chilenos en relación a lo que debieran medir según las normas de la Organización Mundial de la Salud. Se aprecia que los niños que nacieron en 1967 y que por lo tanto tenian 16 años de edad, presentaban de promedio un $7 \%$ de déficit de talla. Desde alli en adelante el déficit va disminuyendo, y los que nacieron en 1978 tienen un déficit de sólo un 2\%. Esto significa que está llegando a la edad escolar un mayor número de niños cuyo crecimiento ha sido normal. La menor diferencia favorece casi siempre a las mujeres. 
en definitiva va creando por más tiempo nuevos recursos que pueden usarse para ayudar a la siguiente generación y así sucesivamente continuar prosperando" (20). Lo logrado hasta ahora hace pensar que en las futuras generaciones el cambio debiera continuar potenciándose. La experiencia chilena parece demostrar que lograr un recurso humano indemne durante los primeros períodos de la vida, es una necesidad ineludible, si se pretende alcanzar el desarrollo y el bienestar para todos.

El costo de la inversión en la prevención de la desnutrición de los primeros años de vida, ha sido altamente rentable y no comparable con ningún otro. Una verdadera revolución es la que logra proteger al niño desde las primeras etapas de su vida. El beneficio comienza en Chile a consolidarse con el desarrollo de una pujante clase media, que durante los últimos años se ha triplicado, pasando a liderar el proceso dentro del conjunto de los países de la región en América Latina (figura 9) (21). En resumen, el chileno promedio de hoy día, ha mejorado significativamente su nivel de vida: vive mas años, con menos enfermedades infectocontagiosas, con una alimentación de mejor calidad, es más alto, más fornido, mental y culturalmente más desarrollado, es más rico, con mayor acceso a bienes y servicios, en relación a lo que fueron sus padres y abuelos.

\section{RESUMEN}

Para prevenir el daño se implantaron exitosas intervenciones, destinada a proteger al niño durante los primeros períodos de la vida. Como resultado, el $98 \%$ de los menores de cinco años en el país, está creciendo y desarrollándose dentro de parámetros normales establecidos por la OMS. En los años sucesivos, el impacto (efecto dominó) fue repercutiendo en edades posteriores, exteriorizándose en el incremento de las demandas educacionales y de salud, que culminaron con un posterior período de rápido desarrollo social y económico. Se concluye que la inversión en la prevención de la desnutrición de los primeros períodos de la vida, ha sido altamente rentable y no comparable con ninguna otra. El ejemplo de lo ocurrido en Chile durante este periodo, puede ser de utilidad para otros países de la región que aún padecen de similares problemas nutricionales y de pobreza.

Palabras clave: daño cerebral, desnutrición, impacto socioeconómico, prevención.

Agradecimientos: Reconocimiento a los académicos e investigadores que entre los años 1952 y 2000 participaron en el desarrollo de las investigaciones básicas, aplicadas y operacionales necesarias, tanto en el Laboratorio de Investigaciones Pediátricas (Hospital Arriarán), como luego en INTA de la Universidad de Chile:

Eduardo Agosín, Marcela Aguayo, María Teresa Aguayo, Cecilia Albala, Guillermo Alead, María de la Luz Alvarez, María Angélica Alliende, Jorge Alvear, Isidora Andraca, José Luis Arias, Magdalena Araya, Juan Alfonso Asenjo, Betty Ávila, Digna Ballester, Ita Barja, Gladys Barrera, Francisco Beas, Teresa Boj, Maruja Bravo, Óscar Brunser, Daniel Bunout, Blanca Curotto, Raquel Burrows, Raúl Cabrera, Bruce Cassels, Carlos Castillo, Iván Contreras, Marta Colombo María Elena Cordero, Verónica Cornejo, Sergio Cornejo, Fanny Cortéz, Miriam Crovetto, Sylvia Cruchet, Bianca Curotto, Patricia Chadud, Saturnino De Pablo, Gonzalo Donoso, Gabriela Duran, Julio Espinoza, Juan Ignacio Egaña, Mireya Fernández, Hernando Flores, Guillermo Figueroa, Carola García, Fernando Garrido, Vivían Gattás, Osvaldo González, Martín Gotteland, Sonia Guerrero, María Teresa Guzmán, Gloria Heresi, Alejandro Hernández, Eva Hertrampf, Sandra Hirsch, Francisco Huneeus, Antonio Infante, Hernán Iturriaga, Daniza Ivanovic, Sergio Jarpa, Gloria Jury, Juliana Kain, Judith King, Ivés Lacassie, Sandra Llaguno, Manuel Llanos, Rubén Leal, Inés López, Alejandro Maccioni, Francisco R. Mardones, Francisco S. Mardones, Norman Merchack, Samuel Middleton, Carlos Muñoz, Santiago Muzzo, Susana Nieto, Fernando Novoa, Manuel Olivares, Sonia Olivares, Sergio Oxman, Berta Otto, Patricio Peirano, Marco Perretta, Margarita Petermann, Ana María Pino, Hernán Pérez, Nelly Pack, Fernando Pizarro, Erna Raimann, Felice Ravenna, Ernesto Ríos, José Riumalló, Claudio

\section{Evolución de las familias en Chile 1990-2011 (\%)}

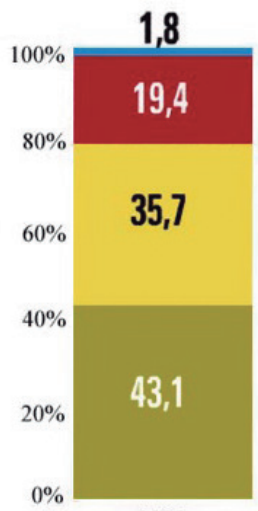

1990
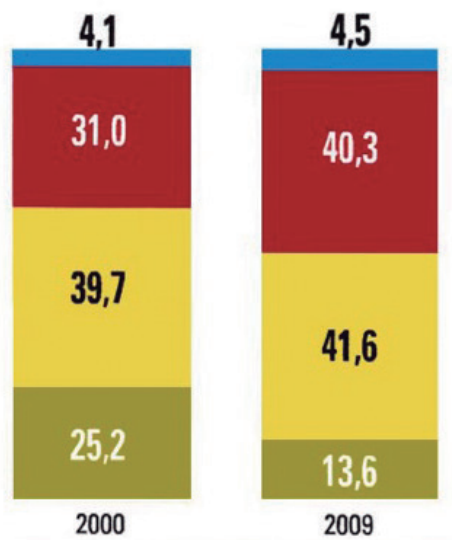

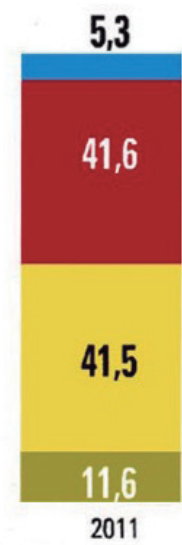

Pobres

Vulnerables Clase alta 
Romo, Ana María Ronco, Mario Rossemblatt, Jaime Rozowski, Samuel Ruíz, María Teresa Saitua, Alfonso Salinas, Ana María Sandino, Liliana Schlesinger, Teresa Segure, Walter Sierralta, Raúl Spada, Hernán Speisky, Giorgio Solimano, Rubén Soto Moyano, Eugenio Spencer, Abraham Stekel, Erika Taucher, Jacobo Tieffenberg, Susana Tisler, Sonia Toro, Irene Truffello, Ricardo Uauy, Guillermo Ugarte, Alfonso Valenzuela, Sergio Valiente, Luis Valladares, Isabel Vial, Ernesto Villa, Fernando Vio, Ennio Vivaldi, Tomás Walter, Enrique Yáñez e Isabel Zacarías.

\section{BIBLIOGRAFÍA}

1. Mönckeberg F. Jaque al Subdesarrollo. Editorial Gabriela Mistral Ltda. Bellavista 0153, Santiago Chile, 1976.

2. Mönckeberg F. Effect of early malnutrition on subsecuent physical and psychological development. En: Malnutrition Lerning and Behavior, Scrimshaw, N and Gordon E. (Ediores). Cambridge, Massachusetts: M.I.T. Press 1968: p 269-78.

3. Mönckeberg F. Integrating national food, nutrition and health policy: The chilian experience. En: Frontiers of $\mathrm{Nu}$ trition and Food Security. Kotler NG.(Editor). Smithsonian Institution Press, 1992.

4. Mönckeberg F, Valiente S. Food and Nutrition Policy in Chile. En: Consejo Nacional para la Alimentación y Nutrición (COMPAN) Santiago Chile 1974.

5. Gonzalez N, Infante A, Schlessinger $C$, and Mönckeberg F. Effectiveness of supplementary feeding programs in Chile. En: Nutrition Intervention Strategies in National Development. Underwood B. (Editora). Academic Press N.Y. 1983: p 103-10.

6. Mönckeberg F. Socioeconomic develpment and nutrtion status: Efficiency of intervention programas. En: Nutrition Intervention Strategies in Development. Underwood B. (Ed) Academic Press N.Y. 1983: 31-40.

7. Mönckeberg F, Riumallo J.: Nutrtion recovery centers: the chilean experience. En: Nutrition Intervention Strategies in Development. Underwood B. (Editora.) Academic Press N.Y. 1983: 189-99

8. Mönckeberg F, y Albino A. Desnutrición "el mal oculto". Inca Editorial Cooperativa de Trabajo Ltda. Mendoza,
Argentina 2004.

9. Brunser O, Reid A, Mönckeberg F, Maccioni A. Jejunal biosies in infant malnutrition with special reference to mitotic index. Pediatrics 1966; 38: 605-12.

10. Schlesinger L, Weimberger J, Figueroa J, Secure, MT, Gonzalez N y Mönckeberg F.: Enviromenntal intervention. En: Underwood B Editores. National Development. New York: Academic Press, 1983; p. 241-53.

11. Ivanovic DM, Leiva BP, Pérez $H$, y cols. Nutritional estaus, brain development and scholastic achivement of children high-school graduates from high and low quotient and socioeconomic status. Br J Nutr. 2002; 87:81-92.

12. Mönckeberg F, Donoso G, Valiente S, Arteaga A. Análisis y comentario de la encuesta nutritiva y de las condiciones de vida en la Provincia de Curicó. Rev Chil Nutr. 1967; 38: 522-35.

13. Ravelli SP, Stein ZA, Susser MV. Obesity in young men after famine exposure in utero and early infancy. N Eng J Med. 1976; 295: 349-53.

14. Barker DJ, Osmond C. Infant mortality, child nutrition and ischaemic hearth disease. Lancet 1986; 1: 1077-81.

15. Mönckeberg F. De la desnutrición a la obesidad. Nutr Salud Bienestar Nestlé 2005: 5:18.

16. Visscher O. Sizing up human variations. Natural Genetics 2008;40:489-91.

17. Mönckeberg F, Donoso G, Valiente S, Arteaga A. Análisis y comentarios de la encuesta nutritiva y de las condiciones de vida de la Provincia de Curicó. Rev Chil Nutr. 1967; 38: 522-35.

18. World Health Organization, Mother/Child Demographic, Health and Nutrition Survey, 2004.

19. Mönckeberg F, Uauy $R$, Cristi MK. El chileno está aumentando de estatura. Creces 1984; Diciembre: $p$ 18-20.

20. Fogel $R$, Floud $R$, Herris B, Choul Hong $S$. The changing body: health, nutrition and human development in Western World since 1700. Cambridge University Press, New York 2011.

21. Marticorena N. Chile triplica su clase media en siete años, liderando el nivel en America Latina. El Mercurio, cuerpo B; Febrero 2013. 